\title{
Intermediate-dose intramuscular methylprednisolone acetate in the treatment of rheumatic disease
}

\author{
JOEL KOVARSKY \\ From the Baylor College of Medicine and the Methodist Hospital, Houston, Texas 77030, USA
}

SUMmARY Treatment with a high-dose, intravenous bolus of methylprednisolone has been reported in numerous cases of rheumatic diseases. The optimal routes of administration, dosage, dosage interval, and other factors are unknown. This report describes uncontrolled clinical observations with a single dose of $320 \mathrm{mg}$ of intramuscular metylprednisolone acetate in patients with rheumatic disease (12 with rheumatoid arthritis, 2 with spondyloarthropathy). This treatment may provide a useful therapeutic adjunct in selected clinical circumstances.

During the past few years increasing use is being made of high-dose, intravenous glucocorticoid (pulse) therapy in various rheumatic diseases. Reports of pulse therapy for disease flares of systemic lupus erythematosus, ${ }^{1}$ rheumatoid arthritis, ${ }^{2}$ and spondyloarthropathy ${ }^{3}$ have all been published. Pulse therapy usually employs $1.0 \mathrm{~g}$ boluses of methylprednisolone sodium succinate given for one to 3 successive days, infused over a time interval ranging from 30 minutes to 2 hours per bolus. Very little information is available on the optimal total dose, the frequency of intermittent pulses, or the effect on the long-term course of the disease.

The purpose of this report is to describe experience with several patients with rheumatic disease (rheumatoid arthritis and spondyloarthoropathy) receiving intermittent intramuscular injections of a single dose $(320 \mathrm{mg})$ of methylprenisolone acetate. This route of administration may offer several advantages over the intravenous route discussed above, and it may be a useful therapeutic adjunct in selected patients with rheumatic diseases.

\section{Methods}

Methylprednisolone acetate suspension (MPA; 80 $\mathrm{mg} / \mathrm{ml}$ ) was administered by bilateral intragluteal injections of $2 \mathrm{ml}(160 \mathrm{mg})$ each. This represented a total dose of $320 \mathrm{mg}$ per administration. There were no clinically apparent side effects other than local bruising at the injection site in any of the patients.

Accepted for publication 1 April 1982.

Correspondence to J. Kovarsky, MD, 6560 Fannin-M.S. 815-S, Houston, Texas 77030, USA.
There was no significant weight gain or induction of cushingoid appearance in any patient.

Twelve patients with definite or classical rheumatoid arthritis and 2 with spondyloarthropathy have received a total of 30 injections over the past year. No patients with American Rheumatism Association (ARA) functional class IV disease present for over 2 months were included. Over $80 \%$ of the injections were followed by dramatic clinical improvement beginning within 36 hours of the injection and lasting at least 2 to 3 weeks. Four brief representative cases are discussed below.

\section{Case reports}

CASE 1

The patient was a 26-year-old blacksmith with a one-year history of radiographically confirmed sacroiliitis. For 6 months prior to his initial visit here he had been largely incapacitated by his low back pain. $\mathrm{He}$ was unable to work half-time and was having substantial sleep disturbance from the pain. Various nonsteroidal drugs, including phenylbutazone and indomethacin, had provided minimal subjective relief and caused recurrent gastrointestinal upset.

He was first seen here in mid-July 1981, given intramuscular MPA, and started on naproxen $375 \mathrm{mg}$ twice a day with food. Within 24 hours his pain had almost entirely cleared, he was able to sleep without difficulty, and he returned to full-time work (actually, double shifts). Two weeks later, because of some recurrence of pain, the intramuscular MPA was repeated, with some additional subjective response, though clearly less dramatic than after the first injection. When last seen, in early October 1981, his only 
complaint was of some mild, intermittent left lateral thigh pain. He was sleeping well, still working double shifts (against persistent advice to the contrary), and taking naproxen regularly.

CASE 2

The patient was a 45-year-old university professor with a 10 -year history of radiographically confirmed spondyloarthropathy. He had a history of 2 separate bouts of uveitis controlled with topical steroids. He was first seen here in mid-March 1981 with a 3-month history of increasing low back and thoracic girdle pains, uncontrolled by $1600 \mathrm{mg}$ per day of ibuprofen in divided doses. He was sleeping poorly and unable to function normally and work because of his pains.

The patient was given intramuscular MPA, and his ibuprofen was changed to sulindac, $200 \mathrm{mg}$ twice a day with food. Within 24 hours his back pain disappeared and his sleeping habits and job function had returned to normal. Except for intermittent minor backaches he has remained stable on sulindac alone for over 7 months, with no further glucocorticoid therapy.

CASE 3

The patient was a 52-year-old housewife with a 20-year history of classical rheumatoid arthritis by ARA criteria. In September 1979 she had a polyarticular flare, and she was begun on $750 \mathrm{mg}$ per day of naproxen in divided doses, and prednisone $5 \mathrm{mg}$ every morning. After 2 weeks she was substantially improved, and remained stable on these drugs until May 1981, when she had a one-month history of steadily progressive pain and swelling involving multiple metacarpophalangeal (MCP) and proximal interphalangeal (PIP) joints, the wrists, elbows, and knees. She could no longer sleep comfortably or do her normal housework. Physical examination confirmed evidence of synovitis at all sites mentioned.

Her naproxen and prednisone were held constant, and she was given intramuscular MPA. Hydroxychloroquine, $200 \mathrm{mg}$ twice a day, was also begun. Within 24 hours virtually all the swelling and pain disappeared. She could sleep comfortably and resumed her housework. She has remained clinically and functionally stable for over 5 months.

\section{CASE 4}

The patient was 50-year-old housewife with a history of definite rheumatoid arthritis by ARA criteria since early 1978. A trial of intramuscular gold therapy in early 1980 was discontinued because of mucocutaneous reaction. Numerous nonsteroidal anti-inflammatory drugs had been discontinued because of gastrointestinal upset, though she could usually tolerate fenoprofen in divided doses of 1800-2400 mg per day. She was also on long-term prednisone, $5 \mathrm{mg}$ every morning.

In mid-March 1981, because of increasing, diffuse MCP and PIP swelling and pain, $200 \mathrm{mg}$ twice a day of hydroxychloroquine was added and intramuscular MPA was given. Her joint swelling and pain disappeared within 1-2 days, but it recurred about 8 weeks later, together with additional swelling of her elbows. Neither a repeat dose of intramuscular MPA nor 2 days (one week after MPA injection) of intravenous pulse methylprednisolone sodium succinate (1 $\mathrm{g}$ per day) relieved her symptoms. Because of progressive physical incapacity the hydroxychloroquine therapy was stopped and azathioprine therapy was initiated.

\section{Discussion}

Treatment with intramuscular methylprednisolone acetate may offer several advantages over higherdose, intravenous pulse therapies. The intramuscular route described here employs a lower total dose, is less expensive, and by-passes the need for intravenous administration. Moreover compliance is assured, and the possibility of gastrointestinal toxicity from oral steroid administration is eliminated. The details of short- and long-term efficacy and the optimal dosage and schedule remain to be clarified for all forms of bolus parenteral therapy in rheumatic diseases. However, persistent use of $1 \mathrm{~g}$ of intravenous methylprednisolone may not be justified. Several of the clinical responses reported here to the lower dose of intramuscular MPA were quite striking.

Although large intravenous boluses of methylprednisolone succinate were initially thought to be relatively free of significant side effects, these have been described..$^{4-7}$ The overall incidence of side effects with intravenous pulse therapy may exceed $50 \%,{ }^{4}$ including occasional reports of sudden death. ${ }^{56}$ Certainly the latter should temper enthusiasm for intravenous pulse therapy in patients with non-life-threatening disease. ${ }^{7}$ Occasional anaphylactic reactions, usually associated with the succinate esters of methylprednisolone, have been reported. ${ }^{\gamma}$ Use of the acetate might circumvent this problem.

The main use of the intramuscular MPA bolus described in this report may be in managing acute or subacute disease flares while simultaneously a more effective long-term conventional therapy is started. Certainly a remission of the disease might occur in some cases without alteration to the treatment or any addition to it. The intramuscular route is suitable for outpatients.

The observations reported here are uncontrolled. 


\section{Kovarsky}

All information was gleaned from a busy private office, so a more detailed, conventional clinical study was not feasible. However, definition of complete or partial remission in rheumatoid arthritis (and similar conditions) is difficult under the best of circumstances. ${ }^{9}$ Although in many of the cases discussed here more than one drug was altered simultaneously, the consistent occurrence of a clinical response within 24-36 hours points to the intramuscular MPA as the agent responsible for it. These preliminary observations warrant further study.

\section{References}

1 Kimberly R P, Lockshin M D, Sherman R L, et al. High-dose intravenous methylprednisolone pulse therapy in systemic lupus erythematosus. Am J Med 1981; 70: 817-24.
2 Liebling M R, Leib E, Mclaughlink B K, et al. Pulse methylprednisolone in rheumatoid arthritis: a double-blind cross-over trial. Ann Intern Med 1981; 94: 21-6.

3 Mintz G, Enriquez R D, Mercado U, et al. Intravenous methylprednisolone pulse therapy in severe ankylosing spondylitis. Arthritis Rheum 1981; 24: 734-6.

4 Garrett R, Paulus H. Complications of methylprednisolone pulse therapy. Arthritis Rheum 1980; 23: 677.

5 Bocanegra T S, Castaneda M O, Espinoza L R, et al. Sudden death after methylprednisolone pulse therapy. Ann Intern Med 1981; 95: 122.

6 Stubbs S S, Morell R M. Intravenous methylprednisolone sodium succinate: adverse reactions reported in association with immunosuppressive therapy. Transplant Proc 1973; 5: 1145-6.

7 Hess E V, Kammen $P$ L. Pulse therapy in rheumatoid arthritis. Ann Intern Med 1981; 94: 128-9.

8 Freedman M D, Schocket A L, Chapel N, Gerber J G. Anaphylaxis after intravenous methylprednisolone administration. JAMA 1981; 245: 607-8.

9 Pinals R S, Masi A T, Larsen R A, et al. Preliminary criteria for clinical remission in rheumatoid arthritis. Arthritis Rheum 1981; 24: 1308-15. 